spent on over-the-counter cough medicines annually, despite limited evidence to support their efficacy. The optimal cough assessment outcome parameter for clinical evaluation of anti-tussive drugs is not known. We investigated the natural course of acute cough with objective and subjective cough assessment tools.

Methods 32 healthy subjects with acute cough due to upper respiratory tract infection (median (IOR) age 30 (32-35) years, 63\% female, mean (SEM) duration of cough 4.2 (0.4) days) were recruited for evaluation. Subjects taking anti-tussives were excluded. Assessments of cough severity included objective 24-h ambulatory cough frequency monitoring with the Leicester Cough Monitor, health related quality of life (HROOL) with the Leicester Cough Questionnaire-Acute (LCQ) and cough severity visual analogue scale (VAS). Assessments were performed at baseline (day 1) and then 4 and 8 days later. Global rating of change questionnaires were completed by patients at each follow-up to assess change in cough severity and determine minimal important difference.

Results At baseline, the median (IOR) LCQ score was 14.7 (10.9-18.3) and mean (SEM) cough VAS was 48 (4) mm, consistent with a severe cough associated with significant impairment in HRQOL. Geometric mean (logSD) 24-h cough frequency $\left(\mathrm{CF}_{24}\right)$ at baseline was 15.0 (0.4) coughs/hr, and at days 5 and 9 were $8.7(0.5)$ and $3.4(0.7)$ coughs/hr respectively. At day 5,3 subjects reported no change, 9 mild, 14 moderate and 6 large change in their cough severity. At day 9, 6 subjects reported mild, 5 moderate and 20 large change in cough severity. The minimal important difference on day 5 for the LCQ was 2.0 , VAS $17 \mathrm{~mm}$ and $\mathrm{CF}_{24} 54 \%$ change from baseline.

Conclusions Acute cough is associated with a significant cough frequency and impairment in HROOL. We suggest clinical trials of anti-tussive drugs should incorporate objective and subjective cough assessment outcome measures. Anti-tussive drugs need to demonstrate a considerable reduction in cough frequency ( $>54 \%$ over a 4-day interval) to establish clinically important efficacy.
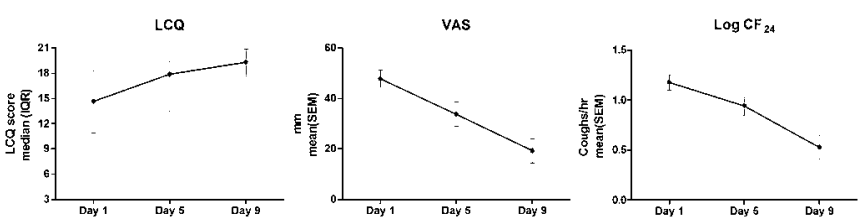

Abstract S144 Figure 1 Change in health related quality of life, cough severity VAS and cough frequency in acute cough.

\section{S144a ENDOGENOUS INHIBITION OF EXPERIMENTALLY INDUCED COUGH IN HEALTHY SUBJECTS}

doi:10.1136/thoraxjnl-2011-201054b.144a

${ }^{1} \mathrm{E} C$ Young, ${ }^{2} \mathrm{~L}$ A Houghton, ${ }^{1} \mathrm{~K} \mathrm{~J}$ Holt, ${ }^{1} \mathrm{~A}$ A Woodcock, ${ }^{1} \mathrm{~J}$ A Smith. ${ }^{1}$ University of Manchester, Manchester, UK; ${ }^{2}$ Mayo Clinic, Jacksonville, Florida, USA

Background The pathophysiology of chronic cough may include peripheral/central sensitisation of afferent pathways and/or a failure of inhibitory pathways. Cough can be voluntarily suppressed in healthy subjects, but the role of endogenous inhibition is unknown. Endogenous inhibitory pain pathways can be activated by applying a painful conditioning stimulus to one body part, to inhibit pain elsewhere, described as "Diffuse Noxious Inhibitory Controls".
Aim To investigate if a painful conditioning stimulus applied to the hand would inhibit cough in healthy subjects.

Methods This was a randomised, 4-way, cross-over study. The EC50 dose of capsaicin was pre-determined (inducing at least $50 \%$ maximal cough frequency) at screening, and subsequently administered at each of the 4 visits ( $>48 \mathrm{~h}$ apart) in 2 blocks ( $1 \mathrm{~h}$ apart) of 4 inhalations (15 s apart), simultaneous with a randomised intervention:

B: Basal-no intervention (both blocks)

W: Warm-hand placed in non-painful $32^{\circ} \mathrm{C}$ water (both blocks) C: Cold-hand placed in painful $10^{\circ} \mathrm{C}$ or non-painful $32^{\circ} \mathrm{C}$ water (randomised order)

S: Suppression-instructed to "try not to cough" or "cough freely" (randomised order) while placing hand in $32^{\circ} \mathrm{C}$ warm water.

Coughs were counted and verified using sound recordings. Urgeto-cough was rated using a Modfied Borg Scale (0-10).

Analysis The between-block change in cough frequency and urge-tocough intensity was compared by intervention using paired t-tests after adjusting for an order-effect. Primary outcome was W versus C. Secondary outcomes were B versus $\mathrm{W}$, and $\mathrm{W}$ versus $\mathrm{S}$.

Results 20 non-smoking healthy subjects [10 male; mean (SD) age 55.05 (14.2) yrs] with normal lung function and median (IOR) EC50 of 15.6 (23.50) $\mu \mathrm{M}$ capsaicin completed the study. Compared to B, $\mathrm{W}$ had no significant effect on cough $(\mathrm{p}=0.623)$ or urge-to-cough $(p=0.285)$. Compared to $W, C$ significantly reduced cough $(p=0.048)$ (Abstract S144a figure 1A) and showed a trend towards a reduction in urge-to-cough $(\mathrm{p}=0.104)$ (Abstract S144a figure 1B). Compared to W, S significantly reduced cough $(\mathrm{p}=0.016)$ (see Abstract S144a figure 1C) but urge-to-cough did not change $(p=0.631)$ (Abstract S144a figure 1D).
A

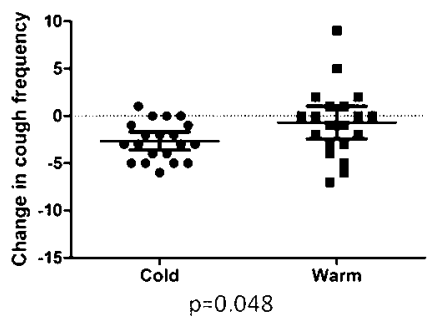

C

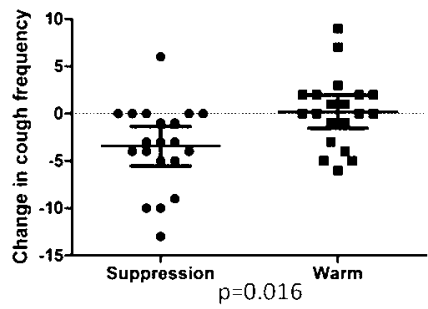

B

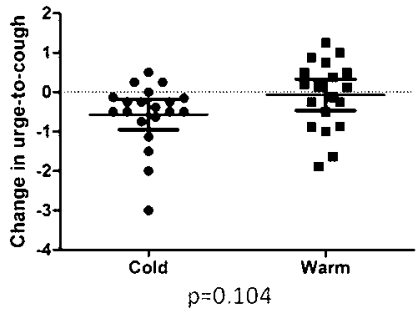

D

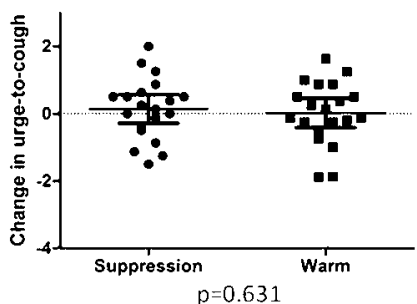

Abstract S144a Figure 1 Change in total cough frequency $(A, C)$ and urge-to-cough intensity $(B, D)$ between blocks. Horizontal lines show mean, error bars $\pm 95 \% \mathrm{Cl}$.

Conclusion Applying a painful stimulus to the hand inhibits cough in healthy subjects, and may be a useful model for measuring endogenous inhibition of coughing. Further studies to investigate whether chronic cough patients demonstrate impaired inhibition using this experimental paradigm are underway. 